\title{
Roms et Tsiganes en Europe méditerranéenne : prégnance et circulation des catégories
}

Roma and Gypsies in the Mediterranean: Categorical Ethnic Distinctions, Rooting and Circulations

Roma y gitanos en el Mediterráneo: distinciones étnicas, enraizamiento y circulaciones

\section{Milena Doytcheva}

\section{OpenEdition}

Journals

Édition électronique

URL : https://journals.openedition.org/remi/7568

DOI : $10.4000 /$ remi.7568

ISSN : $1777-5418$

Traduction(s) :

Roma and Gypsies in the Mediterranean: Circulating Categories, Maintaining Boundaries - URL

https://journals.openedition.org/remi/8478 [en]

Éditeur

Université de Poitiers

\section{Édition imprimée}

Date de publication : 1 mars 2016

Pagination : 165-182

ISBN : 979-10-90426-27-6

ISSN : 0765-0752

\section{Référence électronique}

Milena Doytcheva, «Roms et Tsiganes en Europe méditerranéenne : prégnance et circulation des catégories ", Revue européenne des migrations internationales [En ligne], vol. 32 - $n^{\circ} 1$ | 2016, mis en ligne le 01 mars 2018, consulté le 14 avril 2022. URL : http://journals.openedition.org/remi/7568 ; DOI : https://doi.org/10.4000/remi.7568 


\section{Note de synthèse}

\section{Roms et Tsiganes en Europe méditerranéenne : prégnance et circulation des catégories}

\section{$\ldots$ Milena Doytcheva' ${ }^{1}$}

C'est à une question ancienne et importante pour les sciences sociales que propose de s'attaquer ce recueil d'articles qui est celle des cadrages individuels et plus politiques des démarches d'identification et d'appartenance collective ${ }^{2}$. En prenant pour objet d'étude les situations d'implantation ancienne et la visibilité plus récente, catalysée par les mobilités européennes de populations identifiées comme Roms, Roms etTsiganes ${ }^{3}$, il constitue une invitation à réfléchir à la manière dont des situations historiques d'intégration ont été renouvelées par l'arrivée de nouvelles vagues migratoires. Une entrée particulière est en ce sens privilégiée qui est celle des catégories cognitives et d'action qui ont servi de cadrage à la définition et la politisation de ces enjeux.

En nous focalisant sur les trois principaux pays d'immigration que sont l'Espagne, la France et l'Italie, nous examinons en quoi les mobilités enclenchées dans la période récente, depuis l'Est européen, ont renouvelé ou non des configurations minoritaires plus anciennes. Quelles en ont été les conséquences en matière de stratégies d'identification et de définition collective ? En quoi les catégories ainsi forgées constituent-elles ou non des schémas d'intervention pertinents pour appréhender les dynamiques d'intégration sociale et politique de ceux que les experts identifient comme la " plus grande minorité

\footnotetext{
1 Maîtresse de conférences en sociologie, Université Lille 3, CeRIES (EA 3589), centre de recherche "Individus Épreuves Sociétés ", Domaine universitaire du Pont de Bois, BP 60149, 59653 Villeneuve d'Ascq cedex ; doytcheva.milena@gmail.com

2 Nous nous appuyons ici sur les résultats en cours d'élaboration du projet de recherche "Migrants roms dans I'espace public : (in)visibilités contraintes, mobilisations, habitat " (Ville de Paris, LabexTEPSIS, 2013-2016). Á travers différentes enquêtes portant sur la représentation médiatique, les politiques locales d' " insertion ", la mobilisation associative, il propose d'aborder de manière comparative les stratégies d'intégration différenciées développées vis-à-vis de ces migrants par une pluralité d'acteurs, locaux, nationaux, européens. Une attention particulière est attachée en ce sens à l'étude des dynamiques de "transnationalisation " de la problématique et le retour réflexif qu'elle permet d'opérer sur l'analyse des configurations locales. Le numéro de la revue Confluences en livre un premier ensemble d'hypothèses et pistes d'exploration (Doytcheva, 2015).
}

3 Nous suivons ici l'expression proposée par Liégeois (2009). L'usage de l'italique dans le texte souligne la dimension interactionnelle de ces catégories, construites et endossées dans l'action pour se définir soi-même, ainsi que définir les autres dans des buts d'interaction. Voir aussi infra et la note 4 en particulier. 
européenne "? Le pourtour méditerranéen constitue en ce sens une entrée pertinente, car il semble former en effet un " espace modal " à la fois dans les processus de dispersion historique et les vagues migratoires récentes (Bergeon, 2015).

La synthèse comparative de résultats d'enquêtes sur lesquels le dossier fait retour (portant sur les Gitanos en Espagne, les Romanlar en Turquie, les Roms de Bulgarie, etc.) tend à montrer que les politiques européennes ne sont pas sans quelque incidence sur les processus de construction sociale, nationaux et plus locaux, de leurs figures collectives. Alors même que la littérature insiste sur un " prisme local " dans la perception et la formulation d'une "question rom " lancinante (Sigona, 2005). Faisant fond sur ces rapides remarques préliminaires, nous soumettons à l'épreuve systématique la nécessité méthodologique d'étudier ces problématiques en tenant compte de différentes " échelles de justice " (Fraser, 2008) - locales, nationales, supranationales et européennes. Si l'émergence d'un espace politique supranational a favorisé et créé dans la période récente la possibilité de revendication de droits et d'égalité (Balibar, 2011), elle est sans doute loin d'épuiser le système de persécutions et d'exclusion pluriséculaire de l'antitsiganisme, dont ces populations sont victimes de manière différenciée dans l'espace et dans le temps.

\section{L'émergence de la figure collective des Roms : une perspective européenne}

Dans la période récente, ces préoccupations se formulent d'abord à I'Est, dans les pays d'Europe centrale et orientale qui, au sortir de plusieurs décennies de gouvernement communiste, doivent reconsidérer la coexistence démocratique de différentes collectivités historiques. Dans ce contexte, les objectifs d'une "protection des minorités " sont énoncés à l'endroit d'abord des populations musulmanes. Puis, au milieu de la décennie, ils se déplacent vers celles identifiées comme Roms (Ragaru, 2008 et 2015), victimes nombreuses d'une paupérisation rampante et $d$ 'une marginalisation sociale et économique croissantes. L'identification commune et en théorie générique de Roms prend son essor et s'impose contre les identifications locales traditionnelles et plus usuelles (Tigani, Tsigani, Cingeneler, Zingari, etc.). Ce renouvellement des stratégies identificatoires participe également d'une logique de "destigmatisation " (Pierrot, 2011). En effet, alors que ces dernières désignations constituent des exonymes, I'appellation Rom (Rrom dans certaines graphies et variantes du romani) est réputée au contraire former un endonyme, et s'inscrit clairement, à tout le moins à sa genèse, dans une logique de revendication identitaire et de mobilisation collective.

\section{Saillance d'une dimension culturelle et prégnance d'un ordre racial}

Pour éclairer ces dynamiques d'européanisation et de " transnationalisation " de la problématique, l'usage des ethnonymes et des processus identificatoires constitue en effet une opportunité heuristique. Si, comme le montre Cuche (2008), la catégorisation sociale est indispensable à l'existence de tout groupe social, catégoriser n'est pas neutre. Dans le français courant, le terme 
est implicitement péjoratif, surtout employé à l'endroit de populations fragilisées et démunies (Pierrot, 2011). Dérivé de kategorein, il signifie à la fois " parler contre, accuser, blâmer " et " énoncer, signifier, affirmer ". En usant du pouvoir de révélation exercé par l'objectivation dans le discours, "les catégories "ethniques" ou "régionales", comme les catégories de parenté, instituent une réalité " (Bourdieu, 1980). Cette "réalité " n'est toutefois pas donnée, mais historiquement construite et située dans les sociétés qui les utilisent et rendent légitimes (Desrosières, 1993 ; Martiniello et Simon, 2005). En tant que mise en forme du social et reconnaissance de groupes pouvant légitimement participer à la vie démocratique, éventuellement revendiquer des droits, ces processus constituent un enjeu stratégique de pouvoir dans les sociétés post-migratoires. C'est le pouvoir d'imposer une vision du monde et une représentation perçues comme légitimes, mais aussi le pouvoir de re-signifier des identités assignées, pouvoir souvent nié au minoritaire (Guillaumin, 1972).

Dans I'introduction au dossier (Doytcheva, 2015), nous avons suivi l'expression proposée par Liégeois (2009), de Roms et Tsiganes pour identifier les populations étudiées. Si du pont de vue de l'auteur, ces deux termes ne sont pas synonymes, démultiplier les stratégies d'identification permet de souligner, selon nous, la fluidité et la malléabilité de ces identités et appartenances collectives. Cela permet de combiner en outre une auto et une hétéro-identification, étant entendu aussi que nombre de personnes désignées aujourd'hui comme Roms s'identifient d'abord, y compris dans les pays d'émigration, comme Tsiganes. Partant, notre parti pris est aussi celui de nous éloigner de toute définition "objective " de ces groupes et "identités " collectives, en nous intéressant davantage à la manière dont les labels et distinctions sont construits en situation, par une pluralité d'acteurs ${ }^{4}$.

Dès la fin des années 1960, le Conseil de l'Europe en est un des premiers sur le plan institutionnel. À la faveur d'une série de résolutions, recommandations et rapports, son action peut être caractérisée comme globale : la promotion de la culture, l'enseignement de la langue, l'accès à l'information et à l'éducation y occupent une place importante (Liégeois, 2007). Dans la perspective développée, lesTsiganes ou Gypsies (en anglais) sont aussi une " authentique minorité européenne " qui contribue pleinement à la diversité sur le continent (recommandation 1203 de 1993). La question de leur identification et reconnaissance - collectivités locales versus transnationales et européennes - a été ces dernières années au centre des travaux d'expertise. En 2003, à la suite d'un séminaire dédié Conseil s'engage dans " une harmonisation terminologique " : la dénomination " tsigane " est officiellement proscrite en 2005, à la demande d'organisations de la collectivité, qui y voient une identification exogène, paternaliste et chargée de stéréotypes. À la fin de la décennie, le terme "Roms " s'impose comme désigna-

\footnotetext{
4 Nous nous inscrivons ainsi dans une approche dynamique et relationnelle des identifications et labels ethniques (Barth, 1969), construits et endossés par les acteurs sociaux en situation, pour catégoriser soi-même et les autres dans des buts d'interaction. Dans cette approche, les distinctions particulières sont entendues d'abord, non pas comme recouvrant des identités et entités " objectives ", mais comme le résultat du maintien des frontières ethniques, tracées à la faveur de processus plus larges de stratification et de pouvoir. Voir aussi Martiniello (1995) ; Poutignat et Streiff-Fénart (2008).

5 « Les identités culturelles des Roms, Tsiganes, Gens du voyage et groupes apparentés en Europe ", séminaire organisé au Conseil de I'Europe en septembre 2003.
} 
tion générique et en théorie commune à l'ensemble des collectivités ${ }^{6}$. À noter que ces travaux furent suivis par d'autres acteurs européens, dont de manière notoire la Commission Européenne, I'Agence pour les droits fondamentaux de I'UE (FRA). Afin de combattre les effets d'imposition et d'assignation associés à une catégorisation univoque, des graphies alternatives ont été utilisées : Rroms (avec deux " $\mathrm{rr}$ " comme dans certaines variantes du romani et dans certaines écoles), Rom (sans "s " au pluriel, terme à l'origine invariable); ou encore, la démultiplication des catégories identificatoires, plutôt dans le champ académique, à laquelle nous souscrivons ici : "Roms/Tsiganes ", "Roms etTsiganes ", "Roms et Sintis " en Italie.

Mais, s'il a ouvert de nouveaux espaces de revendication de droits et d'égalité, le développement des institutions européennes et communautaires (EU) aurait produit sur ces questions des " effets contradictoires " (Balibar, 2011). Selon Balibar, si d'un côté I'Europe a conféré à ces populations une identité commune, ou plutôt une catégorisation, cela aurait contribué, d'un autre côté, à un processus de racialisation (cf. aussi infra) des Roms, se voyant ainsi incorporés dans une structure " d'apartheid européen " en voie de constitution. Participe d'un tel malentendu la question des "origines ", fréquemment posée à leur endroit, significative d'une maladroite volonté de valorisation - comme " peuple indo-européen ", venu d'Inde, etc. - alors que la priorité pour les intéressés est d'obtenir d'abord la même légitimité nationale que leurs concitoyens, ou en quelque sorte le droit à l'autochtonie (Pierrot, 2011).

Dans ces analyses, plusieurs aspects caractérisent la situation actuelle : par leurs trajectoires de mobilité et de déterritorialisation, les populations tsiganes focalisent et mettent au jour des préjugés entre nations européennes. Si I'opinion publique française s'émeut peu des politiques de rejet des "Gens du voyage ", elle semble davantage intéressée par des informations sur les persécutions qui se produisent en Italie ou en Hongrie. Les Roms sont une cible idéale pour la cristallisation et le déplacement et de ces sentiments xénophobes intraeuropéens, car ils forment « le type même de la population sans État, résistant à la territorialisation et à I'homogénéisation culturelle " (Balibar, $2011: 141$ ). II ne faut pourtant pas en limiter la définition aux seuls travaux des instances internationales, engagées pour la " protection des minorités".

Comme le montrent en France les recherches d'Asséo (2007), dès 1905, dans les " congrès de police ", émerge une "question tsigane internationale " qui débouche sur la mise en place à l'époque d'une législation discriminatoire dans la plupart des pays européens. Se pose donc la question de ce qui distingue la situation actuelle de " transnationalisation " par rapport à ces autres configurations historiques ${ }^{7}$ ?

6 Selon la définition proposée : "Le terme "Roms" désigne les Roms, les Sintés (Manouches), les Kalés (Gitans) et les groupes de population apparentés en Europe, dont les Voyageurs et les branches orientales (Doms, Loms) ; il englobe la grande diversité des groupes concernés, y compris les personnes qui s'auto-identifient comme "Tsiganes" et celles que I'on désigne comme "Gens du voyage" " (Conseil de I'Europe, 2012).

7 En deçà et au-delà, pourrait-on dire, de la dichotomie affichée des objectifs - ségrégation, traitement spécifique versus inclusion - non moins soupçonnés, nous I'avons vu, de produire des effets convergents. 


\section{Racisme et néolibéralisme : l'exemple des Roms}

Selon les auteurs d'un ouvrage récent consacré à l'examen systématique des liens à l'œuvre entre racisme et démocratie (Fitzgerald et Cook-Martin, 2014), I'affirmation usuelle selon laquelle ces deux concepts sont antithétiques échoue à rendre compte du fait que les démocraties libérales ont été à l'avant-garde de la promotion de politiques racistes et à l'arrière-garde de leur élimination. Mais si le libéralisme a eu davantage affinité avec le tri et la sélection ethnique, ce lien n'est pas pour eux nécessaire au sens de déterminé. Plutôt que de postuler une corrélation, il s'agirait donc d'étudier la manière selon laquelle le phénomène séculaire de l'antitsiganisme entre aujourd'hui en résonance avec les idéologies néolibérale et capitaliste.

L'hypothèse d'une gestion néolibérale de la ville, qui exclue et renvoie à ces marges les plus précaires en constitue une première modalité. Interrogée par différentes contributions (Günes, 2015 ; Sarcinelli, 2015), elle ne reçoit pas toutefois de validation univoque, tant il est vrai en effet que les exemples d'un traitement urbain ségrégatif et excluant sont nombreux et prégnants, pour que la gouvernance actuelle puisse être seule érigée en variable explicative. Dans un essai récent, en suivant les analyses de Michel Feher, Fassin (2014:62) propose une autre interprétation de l'activation actuelle de l'antitsiganisme par le capitalisme néolibéral. Selon sa formulation, si à la faveur des changements politiques intervenus en 2012 en France, il n'y pas eu de rupture dans la gestion politique de la " question rom ", pas plus que dans celle des politiques d'austérité, c'est que les deux " sont l'envers d'une même politique néolibérale " que ces gouvernements de bord différent ont " en partage ".

En reprenant le vocabulaire de Feher, Fassin s'attarde sur " une condition néolibérale " dont le propre est de donner une valeur à tout, où tout est mu par une " aspiration à s'apprécier ". Mais qu'advient-il dans ces conditions de la vie sans valeur, à peine bonne à jeter? La " question rom " symboliserait ainsi, sur le mode de l'exception minoritaire, ce qui pourrait bien être la règle pour la majorité : une " économie du déchet " et une " politique du rebut ". L'auteur en rapproche ensuite l'analyse du concept foucaldien de biopouvoir : "La race, le racisme, c'est la condition d'acceptabilité de la mise à mort dans une société de normalisation ${ }^{8} "$. Le racisme établit une césure à l'intérieur du continuum biologique auquel s'adresse le biopouvoir. Il est à l'origine d'un régime original de biopolitique inventé par la gouvernementalité néolibérale à l'égard des Roms : "Ne pas laisser vivre, sans pour autant faire mourir " (Fassin et al., 2014 : 70). En prenant la douleur comme analyseur politique, Alunni étudie les formes actuelles de ce biopouvoir qui s'exerce à l'endroit des migrants, à travers la médicalisation des campi romi et la mobilisation de politiques humanitaires et de l'urgence en leur direction. Non sans paradoxe, dans ces exemples, la morale humanitaire contribue à un gouvernement sécuritaire (Ticktin, 2011), lorsque " protéger les Roms " permet de "protéger la société des Roms ". Que ce soit en matière de santé ou de "parentalité ", la coprésence entre suspicion et approche compassionnelle constitue une caractéristique structurante et fortement racisante de la relation à ces sujets (Sarcinelli, 2014 ; Alunni, 2015).

8 Michel Foucault, "II faut défendre la société ". Cours au Collège de France 1976, cité par Fassin (2014:68). 
Comme dans ces exemples, les logiques de l'antitsiganisme peuvent ainsi recouper celles de sa prise en charge. À la lumière de la perspective historique et comparative adoptée, leur lien au libéralisme ne saurait être toutefois essentialisé. Tantôt " totalitaires ", tantôt libérales, démocratiques ou post-communistes, ces expressions du racisme entrent en résonance avec des schèmes de perception et de domination plus généraux dans la société. Comme le montre d'ailleurs Ragaru à partir d'une analyse fine des politiques instituées par les pays de départ, plutôt qu'une contradiction ou opposition idéologique de ces multiples cadrages politiques, c'est une logique de sédimentation qui peut être observée dans les pratiques. À une période où les conseillers (ouest)-européens s'emploient à exporter des "bonnes pratiques", a succédé une configuration plus ambiguë dans laquelle I'homogénéisation des savoir-faire a pour pendant la circulation de schèmes "riches en préjugés désormais partagés " (Ragaru, 2008 et 2015).

\section{Minorité transnationale versus locale ?}

À l'intérieur de l'hypothèse néolibérale, des pistes d'interprétation plus spécifiquement françaises soulignent le rôle joué dans ces processus par les instances européennes et internationales : c'est "la fabrique experte de la question rom ", laboratoire selon I'analyse proposée d'une action sociale néolibérale (Olivera, 2011). En fabriquant cette nouvelle catégorie d'intervention, et en attirant sur elle l'attention des opinions publiques, ces organisations auraient de la sorte subséquemment fabriqué "le problème rom ». Elles auraient procédé d'un effet de diversion, leur permettant de supprimer de l'agenda des questions sociales et économiques, les crises à répétition, les politiques d'austérité. La chute du mur de Berlin aurait joué sur ces questions "le même rôle " que l'affaiblissement de I'Empire ottoman au cours du XIXe siècle, selon un argument que nous pouvons rapprocher ici de la thèse hirshmanienne de la futilité 9 .

L'explication du racisme et des discriminations par l'existence d'une différenciation ethnoraciale n'en est pas moins, à nos yeux, problématique. En effet, dans quelle mesure les structures de différenciation sont-elles à l'origine de systèmes de domination et dans quelle mesure en sont-elles le produit ? Comme l'affirment les théories sociologiques du racisme, "contrairement à ce que l'on croit souvent, l'idée de "race" n'est pas ce sur quoi s'ancre logiquement le racisme, mais au contraire ce qu'il produit. " (De Rudder et al., 2000 : 35 sq.). Réaffirmer cette perspective permet de penser dans les exemples étudiés la saillance des processus d'imposition et d'assignation identitaire. Comme l'écrit Phillips (2007) dans un autre domaine, la culturalisation des objets et des pratiques opère parce qu'elle est familière au majoritaire, et non parce qu'elle lui est " exotique " ou étrangère. Les préjugés s'imposent d'autant plus facilement qu'ils entrent en résonance avec des représentations bien installées et disponibles dans l'imaginaire collectif.

De même, les travaux des instances internationales en la matière ne sont pas tous homogènes. Pour le Conseil (CoE) qui s'attache, nous l'avons vu, à la problématique dès la fin des années 1960, les Tsiganes ou Gypsies sont une

9 Selon I'auteur, celle-ci peut se résumer ainsi : " plus ça change, plus c'est la même chose... " (Hirschman, 1991 : 133 sq., pour la traduction française). 
" authentique minorité européenne " qui contribue pleinement à la diversité sur le continent. La Charte européenne des langues régionales ou minoritaires (1992), "La convention-cadre pour la protection des minorités nationales " (1995) constituent à ce titre deux instruments emblématiques de son action, non spécifiques aux Roms. C'est bien plus récemment que l'UE s'est saisie de la question, à travers des cadrages changeants, et comme le montrent quelques premiers travaux, une construction tâtonnante des "problèmes publics roms ". Mais surtout, ces travaux font l'objet d'une "double stratégie européenne " (Doytcheva, 2011).

Alors que la ratification des instruments internationaux fut posée pour les pays d'Europe centrale et orientale en préalable à leur démarche d'adhésion, cette perspective suscita peu d'adhésion, voire fut accueillie avec suspicion dans les pays d'immigration. Il s'y est agi d'interroger "qui produit "le savoir" sur cet objet ? " et " dans quel but ? (Olivera, 2011). Selon cette analyse, "la rhétorique "Rom, peuple victime" "n'est pas nouvelle. Elle émerge au tournant des années 1990 et les premiers pourparlers des pays d'Europe centrale et orientale, candidats à l'adhésion. "L'épistémologie des savoirs " produits dans ces conditions est critiquée (Farget, 2011). Influencée par une approche seulement " objectiviste " dans la définition des populations, elle induirait la cristallisation de représentations essentiellement victimaires qui leur sont associées. Elle concourrait ainsi à développer une vision " palliative " de leur citoyenneté (Thomas, 2010 : 183), c'est-à-dire organisée par d'autres pour eux et le plus souvent sans eux. Celle-ci aurait de surcroît tendance à sur-homogénéiser les situations locales.

Pourtant quelle que soit la diversité des trajectoires et des situations locales, comme l'attestent les différentes contributions au dossier et au-delà, un grand nombre des travaux de la recherche, les populations ainsi identifiées ont en commun une situation durable de discrimination et d'exclusion, souvent adossée au système séculaire de persécution de l'antitsiganisme. Celui-ci peut se définir comme une forme particulière de racisme, caractérisée par la persistance des préjugés sur le (très) long terme, leur caractère systématique, le fait qu'ils soient acceptés par le plus grand nombre, accompagnés d'actes de violence, d'une déshumanisation des individus, de discriminations institutionnelles et systémiques $^{10}$. De ce point de vue, si les notions d' "identité " ou de "communauté " ont été, à juste titre, sujettes à critique pour les définir, celle de minorité, dans un sens sociologique et non pas statistique, semble s'appliquer en particulier ${ }^{11}$.

Si les principes de revendication de droits et d'égalité ont été au cœur des travaux des instances européennes et internationales dans la période d'aprèsguerre, ils semblent pour l'heure dotés d'une capacité insuffisante à innerver les scènes politiques locales, dans le sens à tout le moins d'un renversement des situations historiques de domination et d'exclusion. Les usages mêmes de la catégorie Rom en portent témoignage. Érigée en emblème de ces collectivités de manière endogène, en 1971, à I'occasion du Premier Congrès

10 http://www.ergonetwork.org/ergo-network/advocacy/anti-gypsyism/

11 À savoir, qui " s'inclue dans les processus de classements sociaux, de différenciation et de hiérarchisation sociale, dans une distribution inégalitaire des biens matériels et symboliques "(Simon, 2006 : 152 sq.). 
mondial qui conduit à la formation de I'Union Romani Internationale quelques années plus tard, elle participe clairement même si confidentiellement d'une stratégie de "destigmatisation ". Emprunté à la langue romani, ce mot qui signifie " homme " permettrait de se différencier des autres en s'identifiant aux " hommes par excellence ". Mais, comme le note le linguiste Pierrot, dès lors que le terme le plus générique est " accaparé pour parler de soi ", il peut du point de vue des autres langues, être perçu au contraire comme spécifique et devenir dévalorisant (Pierrot, 2011). Un retournement idéologique s'opère ainsi dans les pratiques et les représentations où les politiques en faveur des Roms peuvent jusqu'à recouper des situations de discrimination directe, sous prétexte de les prendre en charge. Pour paraphraser Philipps, ce qui prend la forme d'un processus d'accommodement de la différence devient une réimposition de la ressemblance.

\section{Modèles nationaux de diversité et migrations roms}

L'examen des politiques nationales et des situations locales en offre une illustration immédiate. En effet, si l'idée d'une " identité rom " est dénoncée comme une construction politique récente, voire comme un " mirage " des " politiques de reconnaissance ", les logiques de racialisation entrent, elles, en résonnance avec des représentations et des structures de domination bien ancrées dans les visions majoritaires et nationales. Nous entendons la notion de racialisation comme le processus d'essentialisation et de naturalisation de différences socialement produites, qu'elles soient fondées sur la culture, une religion, I'histoire ou des traits physiques et phénotypiques ${ }^{12}$. La logique de la pensée raciste étant d'introduire un déterminisme entre ces traits arbitrairement isolés et construits et les conduites individuelles, le propre des attributs assignés sera d'être considérés comme formant une " essence " ou une différence intrinsèque des populations. Le culturalisme y rejoint la biologisation pour enfermer dans une altérité radicale.

En France, Asséo (2007 : 161) souligne « une marginalité historique consubstantielle des Bohémiens ", adossée à une législation dérogatoire et discriminatoire qui se renforce au tournant du XXe siècle, où une " contamination exotique " opère depuis les migrants vers les nationaux. La République a exercé peu d'impact correctif sur ces questions. Plutôt que de combattre les préjugés ancestraux contre les Bohémiens, les républicains les institutionnalisent au contraire à travers diverses mesures destinées d'abord aux " nomades ", puis aux "voyageurs ". La catégorie administrative des "Gens du voyage " qui désigne, sans que les contours soient clairement définis, des populations soumises à un régime dérogatoire au droit commun est instituée par la loi du 3 janvier 1969, en remplacement de celle de "nomades ", utilisée depuis la loi du 16 juillet 1912. Construite par l'extension, à partir du XIXe siècle, d'une catégorie professionnelle qui est celle de "forains " pour englober les populations définies comme tsiganes, "Gens du voyage " forme une catégorie " ethnicoprofessionnelle ", mais aussi " une catégorie discriminatoire légale " (Blum Le Coat et al., 2004 ; Belqasmi, 2015).

12 Pour d'autres éléments de définition, voir par exemple De Rudder et al. (2000: 32). 
La recherche propose d'éclairer ces processus différentiels d'intégration nationale dans une perspective historique et comparative. En nous focalisant sur l'étude des trois principaux pays d'immigration que sont dans la période récente la France, I'Italie et I'Espagne, nous cherchons à éclairer la manière dont les configurations nationales respectives, construites sur le long terme en direction de collectivités locales, ont pu avoir une incidence sur la prise en compte et la gestion politique des migrations récentes. En quoi ont-elles influencé ou non la réception des programmes et des objectifs communautaires (EU) ? Dans une perspective davantage sociologique, comment les vagues migratoires récentes ont-elles redéfini les contours d'une identité ethnique postulée et les stratégies de mobilisation et de revendication collective ?

Les situations française, espagnole et italienne offrent des opportunités heuristiques fécondes pour aborder ces questions. L'on peut schématiquement les typifier autour de trois logiques : une "politique de reconnaissance " du peuple gitano, dans le cas espagnol, politique dans laquelle seront inclus les " nouveaux Roms ", I’Espagne étant souvent présentée, même si de façon un peu superficielle, comme un " modèle d'intégration " en la matière ; une politique également de continuité, dans le cas italien, construite cette fois-ci non pas sur le versant positif d'une reconnaissance revendiquée, mais sur celui négatif du préjugé et d'une assignation commune à la catégorie fortement racisée de "nomades "; enfin, entre les deux, comme de coutume, la situation française se caractérise par le refus théorique d'une régulation publique de l'ethnicité : les résultats d'enquête ne nous invitent pas moins à considérer les processus par lesquels les situations actuelles se trouvent rapportées à des définitions antérieures de la "tsiganité ", mais aussi, et bien souvent, nous le verrons, de la colonialité.

\section{L'exemple espagnol}

La situation espagnole, relativement peu étudiée en France en comparaison avec celle italienne, offre pourtant une perspective et éclairage particulièrement riches et stimulants. En effet, l'Espagne est le pays où le nombre des nouveaux immigrants est-européens est le plus important : situé dans une fourchette allant de $50000^{13}$ à 170000 personnes (alors que c'est un effectif de 20000 qui est fréquemment donné pour la France et qu'un chiffre de 30000 peut être retrouvé pour l'Italie). À la différence des deux autres pays, I'Espagne n'a pas appliqué de régime transitoire pour la période 2007-2014 en direction des nouveaux citoyens de I'Union. Si cela n'a pas suffi à épuiser les situations de difficulté administrative, il reste à évaluer de manière précise comment cette posture a infléchi I'action et les politiques publiques.

En plus de former une destination privilégiée pour les migrations récentes, le pays fut souvent promu, en effet, en " modèle d'intégration " des Gitanos. Dans le contexte d'une autonomie régionale importante, ces enjeux ont trouvé des relais locaux. La Catalogne ou le Pays Basque se sont appuyés par exemple sur les politiques de reconnaissance pour affirmer leur pouvoir législatif. La Catalogne a officiellement reconnu en 2001 " l'identité du peuple gitano et

13 Estimation basse adoptée notamment dans MSSSI (2012 : 12). 
la valeur de sa culture ${ }^{14}$. Comme le montre ici le choix des termes (celui de peuple, plutôt que de population), l'approche adoptée est explicitement « transnationale " et minoritaire. Elle rencontre en cela les orientations développées par les institutions européennes : beaucoup de plans régionaux de promotion du peuple Gitano ont intégré à partir de la deuxième moitié des années 2000 des dispositifs en faveur des "nouveaux Roms" (Magazzini et Piemontese, 2015). Autre trait marquant, l'Espagne semble être le seul pays (des trois ici étudiés) à avoir orienté ces migrants vers les dispositifs de droit commun en matière d'action sociale de proximité. Que ce soit en France ou en Italie, c'est une tendance inverse, à la création de structures ad hoc et de dispositifs spécifiques qui prédomine, étant pour partie à l'origine d'une visibilité et d'une médiatisation plus importantes.

Les travaux de recherche soulignent également le rôle joué par des acteurs de la collectivité, dont des ONG Gitano et pro-Gitano. Alors que ces associations se montrent au départ peu intéressées, elles commencent à se saisir de la problématique au fur et à mesure de sa politisation au plan national et de la mise en place de fonds européens dédiés. Le ciblage des populations s'appuie donc essentiellement sur un relai associatif, comme c'est le cas également de manière notoire en France lorsqu'il s'agit de populations minoritaires autres que tsiganes ${ }^{15}$. Pour ces dernières, en revanche, comme nous le verrons ci-après, aussi bien en Italie qu'en France, la sélection s'effectue aujourd'hui de manière institutionnelle, à travers des dispositifs d'intervention et des structures ad hoc, relevant de logiques exceptionnelles et dérogatoires au droit commun.

La configuration espagnole qui semble avoir fonctionné pour les populations locales montre aujourd'hui ses limites, lorsqu'il s'est agi de l'orienter en direction des "Roms originaires d'autres pays ", selon I'appellation usitée. En effet, d'une part, le relais associatif manque et n'est pas suffisamment puissant pour prendre en compte l'intérêt de groupes autres que les collectivités historiques locales. D'autre part, de plus en plus de praticiens et professionnels sociaux semblent interroger l'opportunité de l'adéquation dressée entre Gitanos et " nouveaux Roms " en matière $d^{\prime}$ action sociale.

Last but not least, l'allégeance à une identité commune, en théorie revendiquée, est fragilisée par le peu de solidarité et de liens inter-communautaires effectifs. Les enjeux catégoriels priment sur une supposée cause commune, soulignant dans les faits la dimension historique dans la construction de ces situations minoritaires (Sayad, 1977) : ancienne et locale pour les uns, plus récente et enracinée dans la migration pour les autres. En Espagne, mais aussi en France et dans d'autres pays d'immigration, si les mobilités récentes réactivent la question d'une identité ethnique, celle-ci ne se pose pas d'emblée et spontanément dans les termes de la solidarité (Roman, 2014 ; Magazzini et Piemontese, 2015). Les ONG Gitano et pro-Gitano craignent en particulier que le travail réalisé, depuis des décennies, de lutte contre les stéréotypes et préjugés ne soit aujourd'hui dégradé par des rapprochements opérés avec ces gitanos

14 Résolution 1046/VI du Parlament de Catalunya " Sobre el reconeixement de la identitat del poble gitano i del valor de la seva Cultura ", du 21/11/2001 et Butlletí Oficial, n² 240.

15 Sur ces questions, nous renvoyons en particulier à nos travaux sur les politiques de la ville qu'il serait trop long d'exposer ici. 
rumanos. D'autant que la représentation de celui que estafa u obra con engaño ${ }^{16}$ est encore aujourd'hui une définition usuelle du Gitan.

\section{Le " nomadisme tsigane "}

L'Espagne n'en est pas moins le pays où la médiatisation sociale et politique d'une "question rom " lancinante, et des politiques d'expulsion et de rejet qui l'accompagnent, fut moins prégnante, alors même que le nombre d'immigrants y est sensiblement supérieur à ceux enregistrés dans les pays voisins. Contrairement à un postulat fréquent, les prescriptions internationales et les normes européennes ne sont donc pas sans incidence, comme le montre l'exemple espagnol, sur les politiques mises en place au niveau local et national. Loin de refléter cependant une transposition linéaire ou, a contrario, une résistance, ces dernières résultent du redéploiement de savoir-faire et de représentations antérieurs à l'intégration européenne que celle-ci a refaçonnés plus qu'elle n'a disqualifiés (Ragaru, 2015).

C'est également ce que montre l'étude de la situation italienne, où la logique de continuité entre les politiques d'hier et d'aujourd'hui est illustrée, sur le versant négatif des stéréotypes et des préjugés, par le recours à la catégorie fortement essentialisée et racisée de " nomades ". Héritière de la pensée raciale du XIXe siècle et de la recherche fasciste, elle implique les idées de l'hérédité de l'asocialité et de l'infériorité raciale des Tsiganes, populations hétérogènes qui ont en commun d'être identifiées comme telles (Sarcinelli, 2014 et 2015). Au lendemain de la Deuxième Guerre mondiale, toutefois, se développe ici aussi un mouvement de solidarité en leur faveur et des initiatives de valorisation et de promotion sociale voient le jour. L'esprit s'en retrouve à partir des années 1980 dans les politiques municipales, attachées à la mise en place d'équipements spécifiques permettant de les accueillir. Pourtant, comme le montre Sarcinelli, ces différentes philosophies n'eurent dans les faits qu'un seul et même effet : la ségrégation et la marginalisation grâce à la création de dispositifs spécifiques (uffici nomadi, aree di sosta per nomadi, campi nomadi). L'ensemble hétérogène des Roms et Sintis, italiens et étrangers, sédentaires ou voyageurs, réfugiés ou migrants, fut alors regroupé sous la même étiquette de " nomades" et soumis au traitement de bureaux spécifiques. Les aires de stationnement furent progressivement transformées (y compris par leurs habitants) jusqu'à devenir des camps permanents (même si la recherche récente tend à nuancer ce constat) (Maestri, 2015 ; Vitale, 2009). Marquée en apparence par une volonté de protection des minorités, cette période a ainsi vu réapparaître l'argument du " nomadisme tsigane ", mobilisé au début du XXe siècle pour justifier la mise à l'écart. Le caractère multiforme des mobilités observées dans la période récente les éloigne pourtant du dit nomadisme, pour les inscrire pleinement dans les logiques contemporaines de migrations transnationales, pendulaires ou circulaires, et d'une "intégration européenne par le bas " (Diminescu et al., 2003 ; Bergeon, 2015).

Après un temps de problématisation latente, ces développements récents en Italie ont toutefois permis de réinvestir "la question rom ", à la fois dans le

16 Littéralement " trompe ou agit avec duperie ", une définition du Gitano encore en vigueur dans le dictionnaire de l'Académie royale. 
champ politique, le débat public, à I'université. Même si la ligne de I'Union en termes $d^{\prime}$ " inclusion des Roms " semble, à première vue, avoir peu à voir avec les politiques sécuritaires ou d'abandon mises en place au plan local, elle n'a pas été sans quelques influences. Le changement de vocabulaire (de "Nomades" à "Roms ") en fait partie. Le traitement différentialiste persiste pourtant, accompagné de formes d'exclusion plus subtiles et discrètes, mais aux effets tout aussi destructeurs. La catégorie de "Rom " dans ces conditions demeure dans une ambivalence entre " racialisation et catégorisation par l'action publique " (Sarcinelli, 2015). Également convoquée sur le long terme, la " raison humanitaire " corrobore ce traitement ségrégatif, en marge des institutions généralistes de santé et de protection sociale (Fassin, 2010 ; Alunni, 2015). C'est là un autre mécanisme de subordination et de racisation insidieuse, en prise avec des dynamiques biopolitiques de gouvernement et de pouvoir.

\section{L'ethnicité républicaine}

Entre la situation italienne et celle de l'Espagne, les politiques françaises semblent occuper une place intermédiaire et par certains aspects ambigüe (Nacu, 2010), en raison entre autres du credo revendiqué de la "cécité aux origines " et d'un refus de l'ethnicité. Dans les faits cependant, elles semblent très proches de celles italiennes, comme l'atteste un nombre important de travaux comparatifs (Legros et Vitale, 2011), des proximités de terminologie et de vocabulaire. Dès lors, c'est bien souvent cet "impensé républicain " de la dimension raciale, en matière de politique comme d'action publique, que dévoilent les résultats de la recherche.

Les modalités de construction symbolique de l'espace, lorsque les préjugés sur des prétendues "spécificités culturelles " viennent conforter des processus de ségrégation urbaine et de marginalisation spatiale, en constituent un premier exemple et piste d'exploration ${ }^{17}$. $\mathrm{D}^{\prime}$ autres formes de " marquage " opèrent dans les représentations, y compris de manière implicite, contribuant à confiner les images de ces migrants dans une altérité essentialisée et radicale (Dalibert et Doytcheva, 2014 ; Fassin et al., 2014).

Les effets de rupture et de continuité dans le traitement politique de la catégorie bureaucratisée des "Gens du voyage " et de celle aujourd'hui des "Roms migrants " en offrent un autre exemple. En effet, si "Gens du voyage " fait l'objet depuis les années 1970 d'un traitement administratif en théorie " desethnicisé ", les populations originaires de l'Est ont été simultanément perçues en tant que groupe et collectivité, processus dans lequel la dimension ethnoculturelle, mais aussi ethnoraciale a occupé une place importante. Alors que le gouvernement français n'a pas souhaité inclure dans sa "Stratégie nationale pour l'intégration des Roms ", Tsiganes français et Gens du voyage, un parallélisme opère dans les pratiques : de manière implicite, comme dans les mesures $d^{\prime}$ accueil et d'accompagnement mises en place ; et parfois explicite, dans les actes de paroles et les interventions politiques. Ces rapprochements involontaires ou amalgames délibérés (Cossée, 2011), indiquent clairement une

17 Comme dans les propos, parmi d'autres, de Manuel Valls, ministre de l'Intérieur, stigmatisant l'incapacité à s'intégrer et un mode de vie "en confrontation " avec la société française dans une interview donnée à France Inter le 24/09/2013 (Dalibert et Doytcheva, 2014). 
logique essentialiste, d'imputation raciale dans un cadre républicain qui en refuse pourtant hautement le principe ${ }^{18}$. Comme l'écrit Belqasmi (2015), qu'il s'agisse du brandissement de la "menace tsigane " ou du procès en inassimilabilité, tout se passe comme si les représentants des autorités publiques prêtaient le flanc aux accusations de racisme anti-Rom pour mieux passer sous silence la discrimination sociale routinière dont les Tsiganes peu argentés, français ou étrangers, font quotidiennement l'expérience.

Les régimes de l'exception et de la dérogation qui informent les interventions auprès des Roms sur la scène locale ne sont pas en effet sans rappeler "les politiques tsiganes ", adossées elles aussi à des statuts dérogatoires et discriminatoires, au tournant du XXe siècle, dans le contexte d'intensification d'un " travail d'État " d'intégration nationale (Nacu, 2010 ; Belqasmi, 2014 et 2015 ; Doytcheva, 2016).

Ces développements nous autorisent à explorer la manière dont les problématiques contemporaines s'inscrivent, plus particulièrement en France, mais également dans d'autres pays de manière différente, dans un continuum historique, ségrégatif et différentialiste, sans être toujours étranger à une certaine " tradition républicaine " (Bessone et al., 2014). Dans les opérations de destruction d'installations " illicites " - et de leur envers que constituent " les villages d'insertion " - dont la chronique émaille l'actualité, tout comme dans les opérations de " résorption des bidonvilles " au début des années 1970, la mise en place d'une intervention publique en matière d'habitat est bien souvent prétexte à un processus de tri des populations, aboutissant à la sélection de quelques familles jugées " aptes à s'intégrer ", et délaissant la plus grosse partie d'indésirables. Si le dispositif des "villages d'insertion " a essaimé ces dernières années en France comme modèle de bonne conduite, il n'est jamais appliqué à d'autres catégories de population. Ces manières de faire, particulièrement tangibles en ce qui concerne "l'accueil " des Roms, témoignent selon notre hypothèse du fait que leur devenir n'est guère perçu aujourd'hui en France dans les termes d'une " option minoritaire " (Balibar, 2011), mais apparaît bien davantage ancré dans le continuum d'un savoir-faire républicain, ségrégatif, mais assimilationniste.

C'est finalement en observant " par le bas " les parcours et les trajectoires des migrants dans cette "zone de mobilités et de turbulences ", que constitue la Méditerranée, qu'il est possible de reconstituer la complexité d'une " géographie du cosmopolitisme ", permettant de penser de nouvelles formes de cohabitation et d'hospitalité (Bergeon, 2015). Les migrations circulaires ou plus classiques, très intenses, soulignent d'après Bergeon, malgré tout, les fluidités territoriales de l'espace européen que les Roms expérimentent pleinement. L'Italie, la France et I'Espagne constituent pour eux des lieux d'installation privilégiés et engendrent de nouvelles relations, tant à l'intérieur de la cellule familiale qu'avec les sociétés environnantes où ils font l'expérience de la " co-présence " avec d'autres communautés migrantes. Des compétences en renouvellement

18 Voyageurs et Roms ont été inclus ensemble dans la première version de la "Stratégie nationale ", réalisée à la demande de la Commission européenne en 2011 par le gouvernement de François Fillon. En 2013, une révision est promise par Jean-Marc Ayrault, mais qui n'a pas véritablement lieu. Difficile de savoir aujourd'hui si le texte de 2011 fait encore référence, alors que le gouvernement socialiste a fait valoir sa volonté de ne pas traiter dans un même document des " Roms " et " Gens du voyage ". 
sont acquises, des liens sont tissés à l'échelle locale, favorisant une insertion plus rapide, l'entretien et l'amplification des réseaux migratoires.

\section{Conclusion}

Dans un ouvrage récent sur les échelles de justice qui invite à repenser l'espace politique dans un monde globalisé, Fraser (2008) identifie le mécadrage (misframing) comme un mécanisme de production de nouvelles formes d'inégalité et d'injustice, liées à un défaut de représentation, entendue ici au sens de " capacité de configuration des problèmes au sein d'arènes politiques appropriées ". Le principe territorial de la souveraineté nationale a vécu et dans un nombre croissant de situations ne permet plus de définir de façon satisfaisante les sujets $d^{\prime}(i n) j u s t i c e$, dont les causes structurelles ne sont pas à territorialiser. L'auteure en appelle en conséquence à un mode de différenciation politique post-territorial. Si les voies de cette différenciation post-territoriale ne sont pas encore tout à fait claires, elle suggère à minima l'application d'un " all-affected principle" (Fraser, $2008: 24)$ : tous ceux affectés par un phénomène peuvent se constituer en sujets de justice, au-delà de la proximité géographique. Ce qui les réunit est leur dépendance commune de cadrages structurels et politiques qui déterminent leurs perspectives existentielles. Les défenseurs des droits humains, les féministes internationaux, les critiques des politiques " d'ajustement structurel " en offrent des exemples.

De manière similaire, dans les exemples que nous avons étudiés, si la diversité des situations locales est grande, l'importance et le caractère systématique des préjugés de l'antitsiganisme ébauchent I'horizon d'une telle cause commune. L'exclusion et la discrimination, plutôt que, ou avec l'identité et les liens communautaires, rendent possible une politique des minorités qui s'ancre non pas sur des spécificités culturelles, mais sur les causes structurelles de marginalisation et d'exclusion sociale. De ce point de vue, si vue de France "la question rom " semble relever d'une invention récente, il s'agit de ne pas oublier comment elle draine avec soi un système d'inégalités "encastrées " et structurantes.

La démarche de comparaison géographique et historique pour laquelle nous avons proposé ici quelques pistes et jalons nous invite tout particulièrement à ce type de réflexion. Notre propos est aussi celui d'un "décentrement " du regard porté sur ces objets : de l'étude de groupes et populations spécifiques, vers les perspectives plus générales d'une sociologie des migrations, des relations interethniques, du racisme et des discriminations. Participe également de ce mouvement I'exploration systématique de différentes arènes politiques et échelles d'intervention. Entre elles circulent, nous l'avons vu, des schémas perceptifs et des catégories d'intervention, mais plutôt que la juxtaposition, c'est une intégration conflictuelle qui les caractérise, tant sur le plan institutionnel, que sur celui des objectifs d'action. Si l'idée d'une " cause commune " émerge dans les travaux d'acteurs collectifs, dont le portrait demeure d'ailleurs à faire avec précision, nous avons besoin de plus de données sur les relations qui se nouent au quotidien entre migrants et populations locales, minorisées ou non, issues ou non de l'immigration. Cette recherche constitue également une invitation dans ce sens. 


\section{Références bibliographiques}

Alunni Lorenzo (2015) La douleur politique. Enjeux du corps malade dans les campi roms de Rome, Confluences Méditerranée, 93, pp. 103-113.

Asséo Henriette (2007) L'invention des " nomades " en Europe au XXe siècle et la nationalisation impossible des Tsiganes, in Gérard Noiriel Éd., L'ldentification. Genèse d'un travail d'État, Paris, Belin, pp. 161-180.

Balibar Étienne (2011) Racisme et politique communautaire : les Roms, Lignes, 34 , pp. $135-144$.

Barth Fredrik (Ed.) (1969) Ethnic groups and boundaries: The social organization of cultural difference, Boston, Little and Brown, $153 \mathrm{p}$.

Belqasmi Mohamed (2015) Entre dissuasion, assistance et activisme : I'accueil ambivalent des migrants roms en France, Confluences Méditerranée, 93, pp. 127-141.

Belqasmi Mohamed (2014) La construction d'une "question tsigane " : entre catégorisations et mobilisations sociales, Migrations société, 152, pp. 49-56.

Bergeon Céline (2015) La Méditerranée comme carrefour des mobilités des migrants roms, Confluences Méditerranée, 93, pp. 39-50.

Bessone Magali, Doytcheva Milena, Duez Jean-Baptiste, Girard Charles and De Latour Sophie G. (2014) Integrating or segregating Roma migrants in France in the name of respect: A spatial analysis of the villages d'insertion, Journal of Urban Affairs, 36 (2), pp. 182-196.

Blum Le Coat Jean-Yves, Catarino Christine et Quiminal Catherine (2004) Les Gens du Voyage : errance et prégnance des catégories, in Anne Gotman Éd., Villes et Hospitalités : les municipalités et leurs étrangers, Paris, MSH, pp. 157-176.

Bourdieu Pierre (1980) L'identité et la représentation. Éléments pour une réflexion critique sur l'idée de région, ARSS, 35, pp. 63-72.

Conseil de I'Europe (2012) Glossaire terminologique raisonné du Conseil de l'Europe sur les questions roms [en ligne] consulté le 22/07/2016. URL : http://www.angvc.fr/pdf/pouvoirs\%20publics/institutions $\% 20$ europeennes/ COE-20120518-Glossaire-Roms.pdf

Cossée Claire (2011) Les-Roms-migrants-et-gens-du-voyage, Lignes, 35, pp. 167-179.

Cuche Denys (2008) Modes de catégorisation et classement socio-ethnique au Pérou, in Frank Alvarez-Pereyre Éd., Catégories et catégorisation : une perspective interdisciplinaire, Paris, SELAF, pp. 35-55.

Dalibert Marion et Doytcheva Milena (2014) Migrants roms dans I'espace public : (in)visibilités contraintes, Migrations Société, 152, pp. 75-90.

De Rudder Véronique, Poiret Christian et Vourc'h François (2000) L'inégalité raciste : I'universalité républicaine à l'épreuve, Paris PUF, 224 p.

Desrosières Alain (1993) La politique des grands nombres. Histoire de la raison statistique, Paris, La Découverte, $462 \mathrm{p}$. 
Diminescu Dana, Ohliger Rainer et Rey Violette (2003) Les circulations migratoires roumaines : une intégration européenne par le bas ?, Cahiers de recherches de la MIRE, 15, pp. 61-69.

Doytcheva Milena (2016) Between infra-right and public hospitality: Ambiguity in local policies towards Roma migrant families in France, IJMBS, 2 (4), pp. 365-381.

Doytcheva Milena (2015) Roms et Tsiganes en Europe méditerranéenne : I'actualité d'une question, Confluences Méditerranée, 93, pp. 9-25.

Doytcheva Milena (2011) Le Multiculturalisme, Paris, La Découverte, 128 p.

Farget Doris (2011) Étude du discours des juges et experts du Conseil de l'Europe sur l'identité Rom, La "question Rom " en Europe aujourd'hui, Tours, 24-25 mars.

Fassin Didier (2010) La raison humanitaire : une histoire morale du temps présent, Paris, Gallimard-Seuil, 368 p.

Fassin Éric, Fouteau Carine, Guichard Serge et Windels Anne (2014) Roms \& riverains : une politique municipale de la race, Paris, La Fabrique, 240 p.

Fitzgerald David S. and Cook-Martin David (2014) Culling the Masses. The Democratic Origins of Racist Immigration Policy in the Americas, Harvard, Harvard University Press, 512 p.

Fraser Nancy (2008) Scales of justice: Reimagining political space in a globalizing world, Cambridge, Polity Press, 224 p.

Guillaumin Colette (1972) L'idéologie raciste. Genèse et langage actuel, Paris, La Haye, Mouton, $378 \mathrm{p}$.

Günes Burcu Ö. (2015) À l'épreuve de la gentrification et de la transformation urbaine, Confluences Méditerranée, 93, pp. 81-90.

Hirschman Alfred O. (1991) The Rhetoric of Reaction: Perversity, Futility, Jeopardy, Harvard, Harvard University Press, 224 p.

Legros Olivier et Vitale Tommaso (Éds.) (2011) Roms migrants en ville, pratiques et politiques en Italie et en France, Géocarrefour, 86/1, [en ligne]. URL : https:// geocarrefour.revues.org/8210.

Liégeois Jean-Pierre (2009) Roms et Tsiganes, Paris, La Découverte, 128 p.

Liégeois Jean-Pierre (2007) Roms en Europe, Strasbourg, Éditions du Conseil de I'Europe, $310 \mathrm{p}$.

Maestri Gaja (2015) The residential segregation of the Roma in Rome: Explaining the persistence of the Roma camps through comparison, Working paper, SemDoc CEE, Paris, Sciences Po, 16 July.

Magazzini Tina et Piemontese Stefano (2015) Modèles de gestion de la diversité en Europe et migrations roms : le cas espagnol, Confluences Méditerranée, 93, pp. 51-62.

Martiniello Marco (1995) L'ethnicité dans les sciences sociales contemporaines, Paris, PUF, $128 \mathrm{p}$.

Martiniello Marco et Simon Patrick (2005) Les enjeux de la catégorisation, Revue Européenne des Migrations Internationales, 21 (2), pp. 2-11. 
Ministerio de Sanidad Servicios Sociales e Igualdad (MSSSI) (2012) Estrategia nacional para la inclusión social de la población gitana en España 2012-2020, Madrid, MSSSI, $70 \mathrm{p}$.

Nacu Alexandra (2010) Les Roms migrants en région parisienne : les dispositifs d'une marginalisation, Revue Européenne des Migrations Internationales, 26 (1), pp. 141-160.

Olivera Martin (2011) La fabrique experte de la " question rom " : multiculturalisme et néolibéralisme imbriqués, Lignes, 34, pp. 104-118.

Phillips Anne (2007) Multiculturalism without culture, Princeton, PUP, 216 p.

Pierrot Alain (2011) Mythe nomade et logique migratoire, Lignes, 34, pp. 52-78.

Poutignat Philippe et Streiff-Fenart Jocelyne (2008) Théories de l'ethnicité, Paris, PUF, $304 \mathrm{p}$.

Ragaru Nadège (2015) Les productions internationales et locales des frontières ethnoculturelles : les Roms de Bulgarie saisis par les institutions, Confluences Méditerranée, 93, pp. 27-37.

Ragaru Nadège (2008) ONG et enjeux minoritaires en Bulgarie : au-delà de I'" importation/exportation " des modèles internationaux, Critique internationale, 40 (3), pp. 27-50.

Roman Raluca (2014) Trans-National Migration and the Issue of "Ethnic" Solidarity: Finnish Roma Elite and Eastern European Roma Migrants in Finland, Ethnicities, 14 (6), pp. 793-810.

Sarcinelli Alice Sophie (2015) La question rom en Italie, entre logiques sécuritaires et logiques humanitaires, Confluences Méditerranée, 93, pp. 91-102.

Sarcinelli Alice Sophie (2014) Protéger, éduquer, exclure. Anthropologie de l'enfance et de la parentalité roms en Italie, Thèse en sociologie, Paris, EHESS.

Sayad Abdelmalek (1977) Les trois " âges " de l'émigration algérienne en France, ARSS, 15 (1), pp. 59-79.

Sigona Nando (2005) Locating "The Gypsy Problem". The Roma in Italy: Stereotyping, Labelling and "Nomad Camps", Journal of Ethnic and Migration Studies, 31 (4), pp. 741-756.

Sigona Nando and Trehan Nidhi (Eds.) (2009) Romani Politics in Contemporary Europe. Poverty, Ethnic Mobilization, and the Neoliberal Order, Palgrave, MacMillan, $309 \mathrm{p}$.

Simon Pierre-Jean (2006) Pour une sociologie des relations interethniques et des minorités, Rennes, PUR, $347 \mathrm{p}$.

Thomas Hélène (2010), Les vulnérables : la démocratie contre les pauvres, Bellecombe-en-Bauge, Éditions du Croquant, 254 p.

Ticktin Miriam (2011) Casualties of care: Immigration and the politics of humanitarianism in France, Berkeley, University of California Press, 312 p.

Vitale Tommaso (Ed.) (2009) Politiche possibili. Abitare le città con i rom ei sinti, Roma, Carocci, 300 p. 


\section{Milena Doytcheva}

\section{Roms et Tsiganes en Europe méditerranéenne : prégnance et circulation des catégories}

L'article s'appuie sur les résultats en cours d'élaboration du projet de recherche "Migrants roms dans I'espace public " (LabExTEPSIS, Ville de Paris). À travers différentes enquêtes sur les processus de politisation (médiatisation, action collective, politiques locales $d^{\prime}$ " insertion "), il propose $d^{\prime}$ examiner les stratégies différenciées d'intégration développées à l'égard de ces migrants par une pluralité d'acteurs. Faisant retour sur les résultats comparatifs du dossier récent que la revue Confluences Méditerranée consacre à la question, nous analysons en situation les tensions entre une logique de "transnationalisation " de la problématique et l'enracinement des configurations nationales et locales concrètes. Une piste est en ce sens privilégiée qui est celle des catégories cognitives et d'action qui président à la définition de ces enjeux et objets d'intervention.

\section{Roma and Gypsies in the Mediterranean: Categorical Ethnic Distinctions, Rooting and Circulations}

The article is based on results under development of the research project "Roma Migrants in the Public Arena"(LabExTEPSIS, Ville de Paris). Drawing on various fieldworks focused on politicization processes (media coverage, collective action, local policies of "inclusion"), it examines in a cross-scalar approach the differentiated integration strategies developed towards these immigrants by a plurality of actors. Through a critical review of the comparative results issued from the fresh release of Confluences Mediterranée journal, we point out some tensions between processes of "transnationalization" and rooted national and local configurations. We thus favour a particular research-path that focuses on entrenchment and mobilities of categorical ethnic ascriptions and identifications.

\section{..̊̊ Roma y gitanos en el Mediterráneo: distinciones étnicas, enraizamiento y circulaciones}

El artículo se basa en los resultados en progreso del proyecto "Migrantes romaníes en el espacio público" (LabExTEPSIS, Ville de Paris). A través de varias encuestas sobre los procesos de politización (mediatización, acción colectiva, políticas locales de «inclusión»), se propone examinar las estrategias de integración diferenciadas desarrolladas con respecto a estos migrantes. Haciendo una revisión crítica de los resultados comparativos del volumen que la revista Confluences Méditerranée ha dedicado a la cuestión, analizamos en particular la tensión entre un proceso de "transnacionalización" del problema y el enraizamiento de las configuraciones nacionales y locales concretas. Privilegiamos de tal modo una pista de investigación que es la de los modos de categorización que gobiernan la definición de estos sujetos y temas de acción. 